\title{
Honeybee rebel workers preferentially respond to high concentrations of sucrose
}

\author{
Karolina Kuszewska, Krzysztof MiLer, Michal Woyciechowski \\ Institute of Environmental Sciences, Jagiellonian University, Krakow, Poland
}

Received 14 October 2018 - Revised 10 February 2019 - Accepted 5 March 2019

\begin{abstract}
The honeybee is one of several eusocial species with a complex set of anatomical, physiological, and behavioural traits that are correlated with foraging preferences. Sensory sensitivity to sucrose is associated with the foraging preferences of workers, with individuals that are less sensitive to sugar preferring to forage for nectar and those that are highly sensitive to sucrose preferring to forage for pollen. In this study, we test this hypothesis by comparing the sucrose responsiveness of genetically similar normal and rebel honeybee workers, which present different foraging preferences. We found that rebels, which prefer to forage for nectar and have a higher reproductive potential than normal workers, are characterised by sucrose sensitivity that is lower than that of normal workers. Moreover, we show that in both rebel and normal workers, sensitivity to sugar in nectar is lower in workers when their ovaries contain more ovarioles. The results confirm the prediction that bees present a foraging preference depending on their response thresholds to sucrose in solution. However, compared with previous studies, our investigation does not support the assumption that reproductive workers are more sensitive to sucrose concentrations in nectar.
\end{abstract}

\section{honeybee / Apis mellifera / rebel workers / sucrose responsiveness / reproduction / social insect}

\section{INTRODUCTION}

Social insects provide some of the most familiar and spectacular examples of polyphenism, where two or more distinct phenotypes are produced by the same genotype (Nijhout 2003; Simpson et al. 2011; Sultan 2000). The processes underlying reproductive and nonreproductive caste polymorphism in eusocial insects are best understood in the honeybee (Apis mellifera) (Cristino et al. 2006; Leimar et al. 2012; Schwander et al. 2010). The expression of the queen or worker phenotype mainly depends on larval nutrition. Small

Electronic supplementary material The online version of this article (https://doi.org/10.1007/s13592-019-00641-8) contains supplementary material, which is available to authorized users.

Corresponding author: K. Kuszewska,

k.kuszewska@uj.edu.pl

Manuscript editor: Monique Gauthier and facultatively sterile workers develop from larvae that were fed small amounts of royal jelly, while large and reproductive queens develop from larvae that were fed large amounts of royal jelly (Winston 1987). The quantity and quality of food provided at the larval stages affect DNA methylation (Kucharski et al. 2008; Elango et al. 2009), gene expression (Corona et al. 1999; Evans and Wheeler 2001), the haemolymph protein composition (Randolt et al. 2008; Begna et al. 2012), and juvenile hormone titres (Barchuk et al. 2002, 2004).

Polyphenism in honeybees is not limited to queen and worker caste determination. Previous studies showed that worker honeybees display a suite of behavioural traits, including the onset of foraging, learning performance, locomotor performance, and sucrose sensitivity, that are correlated with foraging preferences (Tsuruda et al. 2008; Tsuruda and Page 
2009). Sucrose sensitivity, which is a measure of gustatory responsiveness, is an especially interesting trait that demonstrates significant correlations with many anatomical, physiological and behavioural traits (Tsuruda et al. 2008; Tsuruda and Page 2009). The importance of sucrose sensitivity is clear considering that sucrose is the main carbohydrate source for honeybees, provided by plants as a reward for foragers, and that foraging bees can make the decision to collect nectar based on its sugar concentration. Measurements of sucrose sensitivity are simple to perform and are based on the proboscis extension response (PER). Bees extend their proboscis when a droplet of sucrose solution touches the antennae; therefore, it is possible to measure sucrose sensitivity by using a series of increasing concentrations of sucrose solution (Tsuruda et al. 2008; Tsuruda and Page 2009). Previous studies demonstrated that responses to sucrose vary among bees differing in task specialisation, age, foraging preferences and selection lines. The general pattern is that bees responding to lower concentrations of sucrose are more likely to become pollen foragers than nectar foragers (Pankiw and Page Jr 2000; Pankiw et al. 2001). Moreover, previous studies have shown that these foraging behaviours and PERs are associated with ovary size, such that workers with larger ovaries are more likely to collect pollen than are bees with smaller ovaries, which prefer foraging for nectar (Scheiner et al. 2001; Tsuruda et al. 2008).

A sub-caste of honeybee workers called 'rebel workers' was recently discovered, and these workers provide a good opportunity to investigate polyphenism and the PER because these individuals differ from normal workers in reproductive as well as foraging and behavioural traits (Woyciechowski and Kuszewska 2012; Kuszewska et al. 2018a). These rebel workers differ anatomically from normal individuals as follows: they have more ovarioles in the ovary, larger mandibular and Dufour's glands and underdeveloped hypopharyngeal glands (Woyciechowski and Kuszewska 2012; Kuszewska and Woyciechowski 2015). The development of rebel workers is directly connected with the absence of the queen in the nest (Woyciechowski and Kuszewska 2012) and absence queen's mandibular gland pheromone (Woyciechowski et al. 2017) during the unsealed larva stage of workers, which naturally occurs after swarming which is the only natural method of colony multiplication (Winston 1987). Larvae orphaned by four or more days change their life strategy and develop into rebel workers (Kuszewska and Woyciechowski 2015). The evolutionary explanation for this life strategy of honeybee workers is based on the assumption of inclusive fitness theory (Hamilton 1964) and connected with the dramatic drop in relatedness between old-generation workers and the new queen's offspring (Woyciechowski and Kuszewska 2012). Subsequent research has shown that rebel workers are also characterised by longer lives (Kuszewska et al. 2017), and they initiate foraging trips later than normal workers. Moreover, they usually prefer to collect nectar over pollen, and the nectar they collect is of higher volume and more concentrated than is the nectar collected by normal workers (Kuszewska et al. 2018a). According to the results of previous studies (Tsuruda et al. 2008), the higher reproductive potential of rebel workers suggests that these workers should be more sensitive to sucrose concentration, but their foraging preferences yield the opposite expectation. Therefore, the aim of the present study was to examine whether rebel workers differ from normal workers in sucrose sensitivity and whether they are more or less sensitive to sugar. Additionally, we tested how ovary size is associated with sucrose sensitivity both between the two groups of workers (rebel and normal workers) as well as within each group.

\section{MATERIALS AND METHODS}

We performed our experiments in May and June 2015 in an experimental apiary at the Institute of Environmental Sciences of Jagiellonian University. Five queenright honeybee (A. m. carnica) colonies were studied, each consisting of 20,000-40,000 workers. The colonies were randomly selected for the experiments, and the queens, which were not related to each other, were naturally 
inseminated during nuptial flight. A breeding program with the selected lines was not implemented in this experimental apiary. All colonies were treated in the same way, and the experimental design was previously described by Woyciechowski and Kuszewska (2012) as well as in other papers (i.a. Kuszewska et al. 2017, 2018b; Woyciechowski et al. 2017). At the beginning of the experiment, the queen was restricted to two experimental frames to produce eggs of a similar age (day 0 ). Next, the colony was divided into queenright and queenless subunits, and each subunit was contained in one experimental frame (day 3). When all of the worker cells in the experimental frames were sealed (day 12), the subunits were merged, which is why the experimental broods experienced the same conditions during their prepupal and pupal stages. Before the first adult workers emerged (day 21), all the experimental frames were placed in an incubator in the laboratory $\left(34^{\circ} \mathrm{C}, 90 \% \mathrm{RH}\right)$. The workers were marked on the thorax with a spot of paint (Marabu-Brilliant Painter; with 200 from each group and colony marked) and returned to their native colonies. When the marked workers were 7 days old, 50 workers were captured from each group (reared as larvae in the queenright and queenless subunits). The recaptured workers were subjected to an experiment that tested their sucrose responsiveness. Gustatory responsiveness, i.e., responsiveness to water and sucrose solutions of different concentrations, was measured using the methods describe previously by (Tsuruda et al. 2008; Tsuruda and Page 2009; Scheiner et al. 2013). The bees (50 from each group) were placed individually into cylindrical mesh cages. Each bee was chilled in a refrigerator until the first signs of immobility and mounted in a small tube secured with strips of adhesive tape between the head and thorax and over the abdomen. After recovery, each bee was fed up to $10 \mathrm{ml}$ of $10 \%$ sucrose to control for hunger. If a bee was not hungry, it was removed from the test number of bees per group: colony 1 -normal workers: 43, rebel workers: 34 ; colony 2-normal workers: 46 , rebel workers: 35 ; colony 3-normal workers: 15 , rebel workers:
27; colony 4-normal workers: 43, rebel workers: 47; colony 5-normal workers: 49, rebel workers: 48). Next, the bees were assayed for the PER by touching the antennae with a droplet of sucrose solution or water using the techniques described in Tsuruda et al. (2008). Testing began $30-45 \mathrm{~min}$ after the last bee in each group was fed. Bees were assayed using water $(0 \%)$ followed by a concentration series of $0.1,0.3,1,3,10,30$ and $50 \%$ sucrose by weight, corresponding to a logarithmic series of $-1,-0.5,0,0.5,1,1.5$ and 1.7. All bees were lined up, and each bee was tested once at each of the concentrations in sequential order, i.e., all bees were tested at $0.1 \%$ first, and then all were tested at $0.3 \%$. Prior to each sucrose stimulation, we applied water to the antennae to test for water responsiveness as a control. The interstimulus interval was approximately $5 \mathrm{~min}$. Each bee was observed to 'respond' by fully extending its proboscis when a drop of water or sucrose touched each antenna. The assigned response scores (GRS) ranged from 0 (no response to any concentration) to 8 (response to all concentrations). In the next step, all tested bees were frozen pending organ dissection.

We dissected the hypopharyngeal glands (HPGs) and ovarioles of the frozen workers and examined them under a stereomicroscope. The size of each HPG was calculated from the average size of ten acini, which are saclike dilations that compose the compound HPG (square root of the longest $\times$ shortest diameters of five right-gland and five left-gland acini). We also counted the ovarioles in both ovaries (sum) and evaluated ovary development (Woyciechowski and Kuszewska 2012; Kuszewska and Woyciechowski 2015).

Mixed model two-way ANOVAs were used to compare the parameters (ovariole number and hypopharyngeal gland size) between rebel and normal workers, with the experimental group (reared in queenright or queenless conditions) as a fixed effect and colony as a random effect. According to a previous study by Tsuruda et al. (2008), we assigned the rebel and normal bees to two groups categorised by low responsiveness (GRS, 0-3) and high responsiveness (GRS, 4-8) to sucrose. The differences were estimated using a nonparametric Wilcoxon matched pair test for 
normal and rebel workers originating from the same colony. Moreover, to estimate the association between ovariole number and GRS in groups of normal and rebel workers from each colony, we used linear regression. All calculations were done with STATISTICA 13.0.

\section{RESULTS}

Similar to previous studies (Woyciechowski and Kuszewska 2012; Kuszewska and Woyciechowski 2015; Woyciechowski et al. 2017), we found that the workers that developed under queenless conditions had more ovarioles (Figure 1a; Table S1a) and smaller hypopharyngeal glands (Figure 1b; Table S1b) than workers that developed under queenright conditions, confirming that we successfully obtained both normal and rebel workers. Subsequently, we examined the association between ovary size and sucrose responsiveness in 7-dayold normal and rebel workers. We found that rebel workers characterised by higher numbers of ovarioles in the ovary were more likely to respond to higher concentrations of sucrose (GRS, 0-3) than were normal workers, of which more individuals responded to lower sucrose concentrations (GRS, 4-8, Figure 2; Table S2). Moreover, we found that in each colony and each group (normal and rebel workers), the workers with high
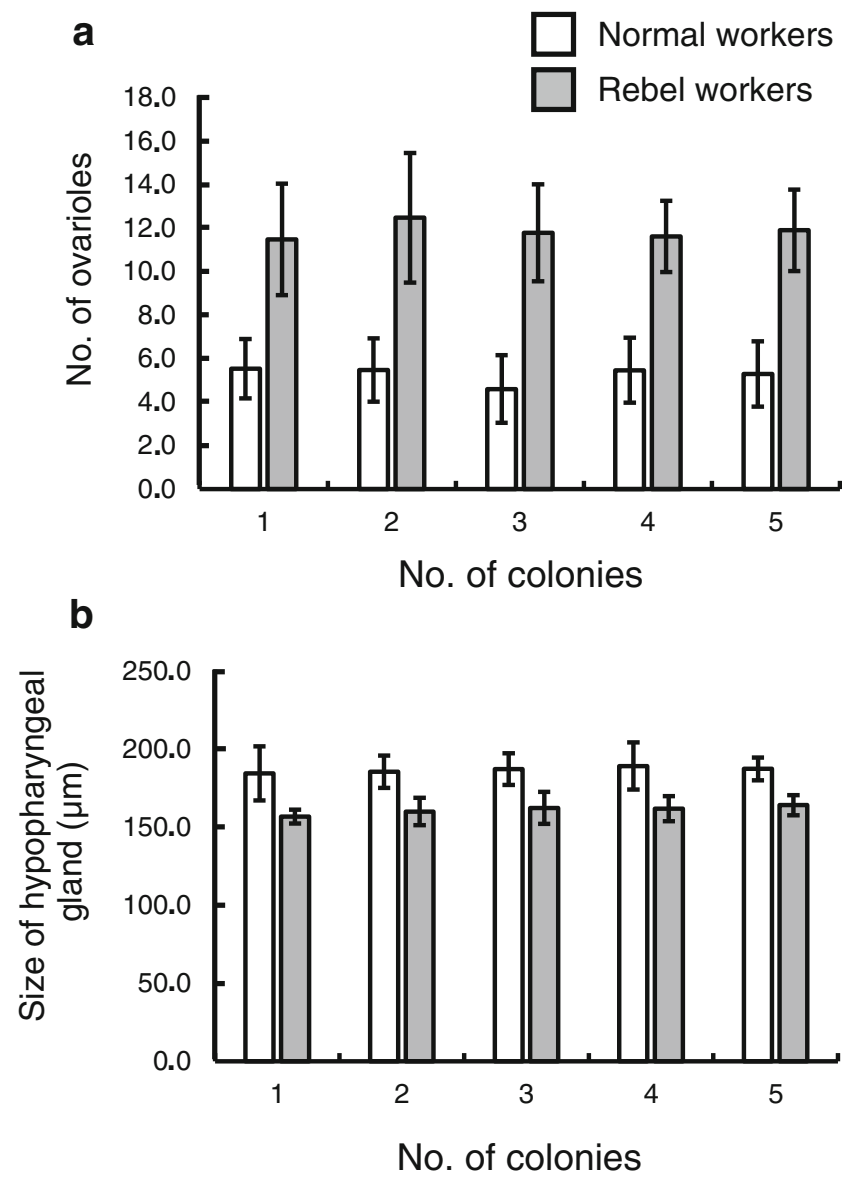

Fig. 1. Anatomical parameters of 7-day-old normal and rebel workers. a Number of ovarioles (mean $\pm \mathrm{SD}$; mixed model two-way ANOVA: $F_{1,4}=816.01, P<0.001$ ). b Size of hypopharyngeal gland (mean $\pm \mathrm{SD}$; mixed model two-way ANOVA: $\left.F_{1,4}=752.94, P<0.001\right)$. 

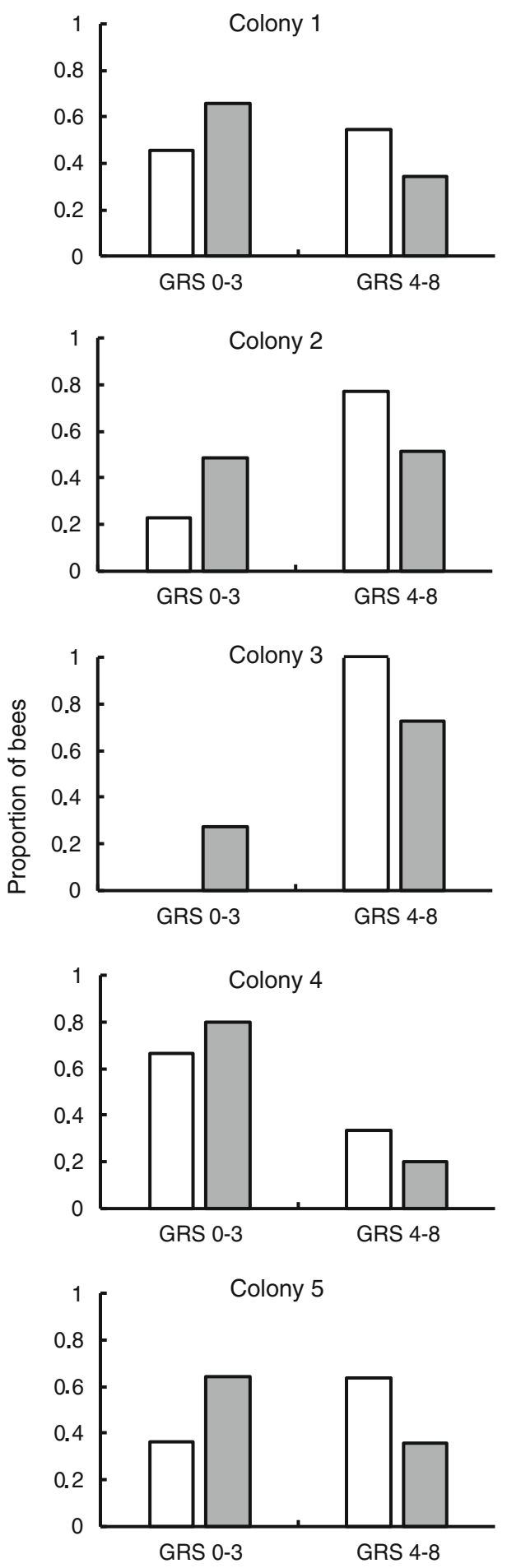

Gustatory response score

Fig. 2. Proportion of normal (empty bars) and rebel workers (grey bars) responding to high (GRS, 0-3) and low (GRS, 4-8) sucrose concentrations (Wilcoxon matched pairs test: $Z=2.03 ; P=0.043$ ). 

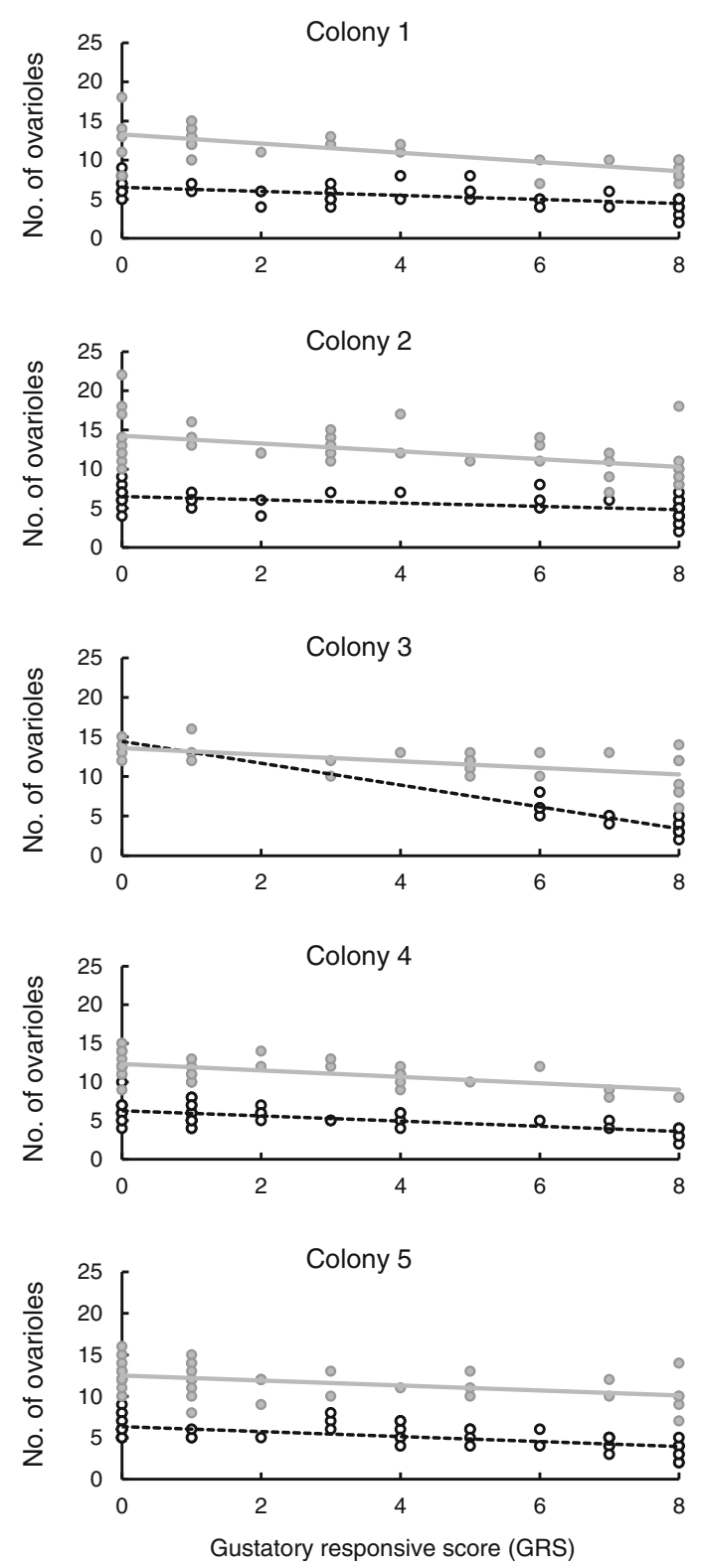

Fig. 3. Relationship between the number of ovarioles and gustatory responsive score in normal (empty circle and dotted black line) and rebel workers (full grey circle and grey line) from five tested colonies. Linear regression. Colony 1: normal workers, $\beta=-0.256, R^{2}=0.3108, F_{1,41}=18.496, P<0.001$; rebel workers, $\beta=-0.586, R^{2}=$ $0.497, F_{1,32}=31.607, P<0.001$. Colony 2 : normal workers, $\beta=-0.2123, R^{2}=0.272, F_{1,44}=16.405, P<0.001$; rebel workers, $\beta=-0.4995, R^{2}=0.276, F_{1,43}=16.393, P<0.001$. Colony 3 : normal workers, $\beta=-1.381, R^{2}=$ $0.219, F_{1,13}=25.886, P<0.001$; rebel workers, $\beta=-0.415, R^{2}=0.322, F_{1,25}=11.873, P=0.002$. Colony 4 : normal workers, $\beta=-0.334, R^{2}=0.374, F_{1,41}=24.545, P<0.001$; rebel workers, $\beta=-0.416, R^{2}=0.329$, $F_{1,45}=22.094, P<0.001$. Colony 5: normal workers, $\beta=-0.299, R^{2}=0.394, F_{1,47}=30.551, P<0.001$; rebel workers, $\beta=-0.299, R^{2}=0.192, F_{1,46}=30.551, P=0.002$.

sucrose responsiveness (GRS, 4-8) were characterised by a lower number of ovarioles than were those with low sucrose responsiveness (GRS, 0-3; Figure 3). 


\section{DISCUSSION}

As we predicted, our results showed that rebel workers, which prefer to collect nectar over pollen and start foraging later in life than normal workers (Kuszewska et al. 2018a), respond to higher sucrose concentrations than do normal workers. This result is in accordance with previous studies that found that nectar foragers respond to higher sucrose concentrations compared with pollen foragers (Pankiw and Page Jr 2000; Pankiw et al. 2001). Bees with high sucrose responsiveness (i.e., pollen foragers) also initiate foraging earlier in life than do those that are less responsive to sucrose (i.e., nectar foragers), and the former have been shown to collect nectar with lower sugar concentrations (Tsuruda and Page 2009). Moreover, studies have shown that among nectar foragers, the individuals that are more responsive to sucrose collect nectar of sugar concentrations lower than those of nectar collected by individuals that respond preferentially to higher concentrations (Pankiw et al. 2001; Pankiw 2003). Because the sucrose responsiveness of young bees is correlated with their foraging behaviour in the future (Pankiw and Page Jr 2000), this connection between sucrose responsiveness and foraging behaviour could have evolved as a protective adaptation against collecting poor-quality nectar (Scheiner et al. 2004). Our results are consistent with this suggestion because rebels that are less sensitive to sucrose in solution prefer to collect nectar with higher sucrose concentrations (Kuszewska et al. 2018a).

The physiological basis for the correlation between sucrose responsiveness and foraging behaviour remains unclear. It has been suggested that this correlation is a consequence of changes in the titres of vitellogenin (Tsuruda et al. 2008), which is a conserved yolk precursor protein. The level of vitellogenin in the bee body is directly related to the number of ovarioles in the ovary, and it is also believed that vitellogenin titres are connected with foraging preferences. These connections among physiology, foraging preferences, reproductive potential of workers and their sucrose responsiveness is referred to as the reproductive ground plan hypothesis (RGPH) (Amdam and Norberg 2004; Amdam et al. 2006; Nelson et al. 2007). According to the RGPH, workers with higher numbers of ovarioles in their ovaries, a characteristic associated with increased productivity, initiate foraging earlier in life, are more likely to present a bias in foraging towards high-protein types of food (e.g., pollen in bees), and are more sucrose sensitive compared with workers with fewer ovarioles (Page et al. 2006; Siegel et al. 2012; Ihle et al. 2014). Previous studies of two honeybee lines selected for high and low pollen hoarding have confirmed this suggestion by showing that high pollen hoarding bees with more ovarioles in their ovaries not only prefer to collect pollen but are also more sensitive to sucrose than bees with fewer ovarioles (Pankiw et al. 2001; Amdam and Norberg 2004; Scheiner et al. 2004; Amdam et al. 2006; Tsuruda et al. 2008). However, other research has focused on a strain of anarchistic bees (a strain selected for abnormal reproductive behaviour) showed a weakness in the RGPH because more reproductive anarchistic bees failed to show a bias towards collecting pollen (Oldroyd and Beekman 2008). Our results also suggest flaws in the RGPH, and although they indicate that foraging preferences are connected with sucrose sensitivity, the relationship between ovarioles and sucrose sensitivity was inconsistent with the predictions of the RGPH because rebel workers, which are characterised by more ovarioles, are less sensitive to sucrose concentrations in nectar than normal workers (Figure 2). Moreover, we also showed that sensitivity to sucrose was negatively correlated with the number of ovarioles in every tested group (normal and rebel workers in five colonies; Figure 3). This result suggests that the relationship between ovariole number and sucrose responsiveness is not as simple as previously thought and that there is likely another physiological mechanism that influences the relationship between foraging behaviour and sucrose sensitivity (Kuszewska et al. 2018a).

In summary, our results support the hypothesis that sensory responses to sucrose concentration are correlated with the foraging behaviour of bees. Individuals with higher sucrose sensitivity prefer to forage for pollen, whereas bees with lower sensitivity prefer to forage for nectar. Moreover, we showed that although the physiological relationships between reproductive potential and sucrose responsiveness and between reproductive potential and foraging preferences are unclear, they may depend on many factors, such as environmental conditions or genetics. 


\section{AUTHOR CONTRIBUTIONS}

Conceived and designed the experiments: KK. Performed the experiments: KK, KM. Analysed the data: KK. Contributed reagents/materials/ analysis tools: $\mathrm{KK}, \mathrm{MW}$. Wrote the paper: $\mathrm{KK}$, MW, KM.Funding information

This study was funded by the National Science Centre (NCN), Poland (grant 2014/13/B/NZ8/ 04705), and Jagiellonian University (grant DS/ BiNoZ/INoŚ/761/15-18). We thank American Journal Experts for language editing (Certificate Verification Key: 2AFA-F0DC-18D0-C1E5$\mathrm{C} 8 \mathrm{~A})$ and two anonymous reviewers.

\section{COMPLIANCE WITH ETHICAL STANDARDS}

Competing interests The author(s) declare that they have no competing interests.

\section{Les ouvrières rebelles répondent de préférence aux fortes concentrations de saccharose}

\begin{abstract}
Abeille domestique / Apis mellifera / ouvrières rebelles / réactivité au saccharose / reproduction / insecte social
\end{abstract}

\section{Rebellierende Arbeiterinnen der Honigbiene bevorzugen hohe Sacharosekonzentrationen}

\section{Honigbiene / Apis mellifera / Rebellierende Arbeiterinnen / Sacharosesensitivität / Reproduktion / soziales Insekt}

\section{OPEN ACCESS}

This article is distributed under the terms of the Creative Commons Attribution 4.0 International License (http://creativecommons.org/licenses/by/4.0/), which permits unrestricted use, distribution, and reproduction in any medium, provided you give appropriate credit to the original author(s) and the source, provide a link to the Creative Commons license, and indicate if changes were made.

\section{REFERENCES}

Amdam G V, Norberg K (2004) Reproductive ground plan may mediate colony-level selection effects on individual foraging behavior in honey bees. Proc. Natl. Acad. Sci. U. S. A. $101: 11350-11355$

Amdam G V, Csondes A, Fondrk MK, Page RE (2006) Complex social behaviour derived from maternal reproductive traits. Nature $439: 76-78$. doi: https://doi. org/10.1038/nature04340

Barchuk A, Bitondi M, Simões Z (2002) Effects of juvenile hormone and ecdysone on the timing of vitellogenin appearance in hemolymph of queen and worker pupae of Apis mellifera. J. Insect Sci. $2: 1-8$

Barchuk A, Maleszka R, Simões Z (2004) Apis mellifera ultraspiracle : cDNA sequence and rapid up-regulation by juvenile hormone. Insect Mol. Biol. 13 :459-467

Begna D, Han B, Feng M (2012) Differential expressions of nuclear proteomes between honeybee (Apis mellifera L.) queen and worker larvae: A deep insight into caste pathway decisions. J. Proteome Res. 11 :1317-1329. doi: https://doi.org/10.1021/pr200974 a

Corona M, Estrada E, Zurita M (1999) Differential expression of mitochondrial genes between queens and workers during caste determination in the honeybee Apis mellifera. J. Exp. Biol. 202 :929-938

Cristino AS, Nunes FMF, Lobo CH, et al (2006) Caste development and reproduction: a genome-wide analysis of hallmarks of insect eusociality. Insect Mol. Biol. 15 :703-714. doi: https://doi.org/10.1111/j.13652583.2006.00696.x

Elango N, Hunt B, Goodisman M, Soojin VY (2009) DNA methylation is widespread and associated with differential gene expression in castes of the honeybee, Apis mellifera. Proc. Natl. Acad. Sci. U. S. A. 106:1120611211. doi: https://doi.org/10.1073/pnas.0900301106

Evans JD, Wheeler DE (2001) Expression profiles during honeybee caste determination. Genome Biol. 2 :RESEARCH0001. doi: https://doi.org/10.1186/gb-20002-1-research0001

Hamilton WD (1964) The genetical evolution of social behaviour I-II. J. Theor. Biol. $7: 1-52$

Ihle KE, Baker NA, Amdam GV (2014) Insulin-like peptide response to nutritional input in honey bee workers. J. Insect Physiol. doi: https://doi.org/10.1016/j. jinsphys.2014.05.026

Kucharski R, Maleszka J, Foret S, Maleszka R (2008) Nutritional control of reproductive status in honeybees via DNA methylation. Science $319: 1827-1830$. doi: https://doi.org/10.1126/science.1153069

Kuszewska K, Woyciechowski M (2015) Age at which larvae are orphaned determines their development into typical or rebel workers in the honeybee (Apis mellifera L.). PLoS One 10 :e0123404

Kuszewska K, Miler K, Rojek W, Woyciechowski M (2017) Honeybee workers with higher reproductive 
potential live longer lives. Exp. Gerontol. 98:8-12. doi: https://doi.org/10.1016/j.exger.2017.08.022

Kuszewska K, Miler K, Woyciechowski M (2018a) Honeybee rebel workers invest less in risky foraging than normal workers. Sci. Rep. 8:9459. doi: https://doi. org/10.1038/s41598-018-27844-w

Kuszewska K, Wącławska A, Woyciechowski M (2018b) Reproduction of rebel workers in honeybee (Apis mellifera) colonies. Apidologie 49:162-171. doi: https://doi.org/10.1007/s13592-017-0537-z

Leimar O, Hartfelder K, Laubichler MD, Page RE (2012) Development and evolution of caste dimorphism in honeybees - a modeling approach. Ecol. Evol. 2 :3098-109. doi: https://doi.org/10.1002/ece3.414

Nelson CM, Ihle KE, Fondrk MK, et al (2007) The gene vitellogenin has multiple coordinating effects on social organization. PLoS Biol. 5 :e62. doi: https://doi. org/10.1371/journal.pbio.0050062

Nijhout HF (2003) Development and evolution of adaptive polyphenisms. Evol. Dev. 5:9-18

Oldroyd BP, Beekman M (2008) Effects of selection for honey bee worker reproduction on foraging traits. PLoS Biol. 6 :e56. doi: https://doi.org/10.1371/journal.pbio.0060056

Page RE, Scheiner R, Erber J, Amdam G V (2006) 8. The development and evolution of division of labor and foraging specialization in a social insect (Apis mellifera L.). Curr. Top. Dev. Biol. 74 :253-86. doi: https://doi.org/10.1016/S0070-2153(06)74008-X

Pankiw T (2003) Directional change in a suite of foraging behaviors in tropical and temperate evolved honey bees (Apis mellifera L.). Behav. Ecol. Sociobiol. 54 :458-464. doi: https://doi.org/10.1007/s00265003-0640-1

Pankiw T, Page Jr RE (2000) Response thresholds to sucrose predict foraging division of labor in honeybees. Behav. Ecol. Sociobiol. 47 :265-267. doi: https://doi. org/10.1007/s002650050664

Pankiw T, Waddington KD, Page RE (2001) Modulation of sucrose response thresholds in honey bees (Apis mellifera L.): Influence of genotype, feeding, and foraging experience. J. Comp. Physiol. A Sensory, Neural, Behav. Physiol. 187:293-301. doi: https://doi. org/10.1007/s003590100201

Randolt K, Gimple O, Geissendorfer J, et al (2008) Immune-related proteins induced in the hemolymph after aseptic and septic injury differ in honey bee worker larvae and adults. Arch. Insect Biochem. Physiol. 69:155-167. doi: https://doi.org/10.1002 /arch.20269
Scheiner R, Page RE, Erber J (2001) The effects of genotype, foraging role, and sucrose responsiveness on the tactile learning performance of honey bees (Apis mellifera L.). Neurobiol. Learn. Mem. 76:138-50. doi: https://doi.org/10.1006/nlme.2000.3996

Scheiner R, Page RE, Erber J (2004) Sucrose responsiveness and behavioral plasticity in honey bees (Apis mellifera). Apidologie 35:133-142. doi: https://doi. org/10.1051/apido

Scheiner R, Abramson CI, Brodschneider R, et al (2013) Standard methods for behavioural studies of Apis mellifera. J. Apic. Res. 52 :1-58. doi: https://doi. org/10.3896/IBRA.1.52.4.04

Schwander T, Lo N, Beekman M, et al (2010) Nature versus nurture in social insect caste differentiation. Trends Ecol. Evol. 25 :275-282. doi: https://doi. org/10.1016/j.tree.2009.12.001

Siegel AJ, Freedman C, Page RE (2012) Ovarian control of nectar collection in the honey bee (Apis mellifera). PLoS One 7 :e33465. doi: https://doi.org/10.1371/journal.pone. 0033465

Simpson SJ, Sword GA, Lo N (2011) Polyphenism in insects. Curr. Biol. 21 :R738-R749. doi: https://doi. org/10.1016/j.cub.2011.06.006

Sultan S (2000) Phenotypic plasticity for plant development, function and life history. Trends Pant. Sci. $5: 537-542$

Tsuruda JM, Page RE (2009) The effects of foraging role and genotype on light and sucrose responsiveness in honey bees (Apis mellifera L.). Behav. Brain Res. 205 :132-137. doi: https://doi.org/10.1016/j. bbr.2009.07.022

Tsuruda JM, Amdam G V, Page RE (2008) Sensory response system of social behavior tied to female reproductive traits. PLoS One 3 :e3397. doi: https://doi. org/10.1371/journal.pone.0003397

Winston M (1987) The biology of honeybee. Harvard University Press, Cambridge, Massachusetts

Woyciechowski M, Kuszewska K (2012) Swarming generates rebel workers in honeybees. Curr. Biol. 22 :707711. doi: https://doi.org/10.1016/j.cub.2012.02.063

Woyciechowski M, Kuszewska K, Pitorak J, Kierat J (2017) Honeybee worker larvae perceive queen pheromones in their food. Apidologie 48:144-149. doi: https://doi.org/10.1007/s1359201604591

Publisher's note Springer Nature remains neutral with regard to jurisdictional claims in published maps and institutional affiliations. 